\title{
Clinical Case Discussion
}

\section{Calculus anuria in a spina bifida patient, who had solitary functioning kidney and recurrent renal calculi}

\author{
S Vaidyanathan*,1, BM Soni ${ }^{1}$, J-J Wyndaele ${ }^{2}$, AZ Buczynski ${ }^{3}$, E Iwatsubo ${ }^{4}$, M Stoehrer $^{5}$, H Madersbacher ${ }^{6}$, \\ R Peschel ${ }^{6}$, G Singh ${ }^{1}$, JWH Watt ${ }^{1}$, PL Hughes ${ }^{7}$ and P Sett ${ }^{1}$ \\ ${ }^{1}$ Regional Spinal Injuries Centre, District General Hospital, Southport, UK; ${ }^{2}$ Centrum Urologische Revalidatie, \\ Universitair Ziekenhuis Antwerpen, Edegem, Antwerpen, Belgium; ${ }^{3}$ Metropolitan Rehabilitation Centre, Department \\ of Neurourology, Konstancin, Poland; ${ }^{4}$ Kyushu University School of Medicine, LWC Spinal Injuries Centre, Igisu, \\ Iizuka, 820-8508, Japan; ${ }^{5}$ Urological Department, Berufsgenossenschaftliches, Murmau, Germany; ${ }^{6}$ University \\ Klinik Innsbruck, Anichstraße 35, A-6020 Innsbruck, Austria; ${ }^{7}$ Department of Radiology, District General \\ Hospital, Southport, UK
}

Study design: Clinical case report with comments by colleagues from Austria, Belgium, Germany, Japan, and Poland.

Objectives: To discuss challenges in the management of spinal bifida patients, who have marked kyphoscoliosis and no vascular access.

Setting: Regional Spinal Injuries Centre, Southport, UK.

Methods: A female patient, who was born with spina bifida, paraplegia and solitary right kidney, had undergone ileal loop urinary diversion. Renal calculi were noted in 1986. Percutaneous nephrostolithotomy was performed in 1989 and there was no residual stone fragment. However, she developed recurrence of calculi in the lower pole of the right kidney in 1991. Intravenous urography, performed in 1995, revealed right staghorn calculus and hydronephrosis. Chest X-ray showed markedly restricted lung volume due to severe kyphoscoliosis. In 2000, she was declared unsuitable for anaesthesia due to a lack of venous access and a high likelihood of difficulty in weaning off the ventilator in the postoperative period. In June 2002, she developed anuria (urine output $=18 \mathrm{ml} / 24 \mathrm{~h}$ ) due to ball-valve-type obstruction by a renal stone at the ureteropelvic junction. Urea: $14.4 \mathrm{mmol} / 1$; creatinine: $236 \mu \mathrm{l} / 1$. Ultrasound showed right hydronephrosis. Percutaneous nephrostomy was performed.

Results: Following relief of urinary tract obstruction, there was postobstructive diuresis $(3765 \mathrm{ml} / 24 \mathrm{~h})$. However, the patient expired 19 days later due to progressive respiratory failure. Conclusion: In this spina bifida patient, who had reached the age of 35 years, severe kyphoscoliosis and lack of vascular access presented insurmountable challenges to implement the desired surgical procedure for removal of stones from a solitary kidney.

Spinal Cord (2004) 42, 7-13. doi:10.1038/sj.sc.3101545

Keywords: spina bifida; anuria; kidney calculi

\section{Introduction}

A retrospective review of 327 patients with neural tube defects showed that there is a higher incidence of nephrolithiasis in patients with neural tube defects than in the general population, and the risk of stone recurrence is also elevated. ${ }^{1}$ Renal stones were noted in $10.7 \%$ of those 12 years old or older. Interventional management of the stones in these patients was associated with $87 \%$ recurrence rates after intervention. Rege $e t a l^{2}$ observed that children with myelomeningocoele, who had ileal conduit urinary diversion, had an

*Correspondence: S Vaidyanathan, Regional Spinal Injuries Centre, District General Hospital, Town Lane, Southport PR8 6PN, UK increased risk of renal calculus formation. Apart from a high incidence of nephrolithiasis, the prevalence of renal parenchymal damage is also high in patients with spinal bifida. In all, $19.4 \%$ of the patients attending a multidisciplinary spina bifida clinic had renal parenchymal damage. ${ }^{3}$ We present a case of calculus anuria in a 35-year-old female patient with spina bifida, who had severe kyphoscoliosis and almost no vascular access.

\section{Case history}

A 35-year-old female patient presented in 2002 with a history of not passing urine for approximately $24 \mathrm{~h}$. The 
day before presentation, she had developed pain in the stomach and back. She had not passed urine from the urostomy. On the day of attending the hospital, she was noticed to be shivering and breathless. Past history included operations for hydrocephalus, spinal fixation, and renal stone. She had a solitary right kidney draining into an ileal loop urostomy.

Percutaneous nephrostolithotomy had previously been performed in 1989, when she was 22 years old. The initial puncture was unsuccessful, but a second puncture was made further laterally and the mid-pole calyx was accessed. The track was dilated and lithotripsy of intrapelvic stone was performed. Rescreening showed solitary lower pole calculus remaining, which was inaccessible from within. Therefore, another track was established to reach the lower pole calyx. Electrohydraulic lithotripsy was performed. The patient was absolutely free of renal stones at the end of the procedure.

In 1991, a routine intravenous urography (IVU) showed opaque calculi in the lower pole of right kidney, which was functioning. In 1995, a routine IVU showed right staghorn calculus (Figure 1) and hydronephrosis. Marked thoraco-lumbar scoliosis was noted. In 1997, a routine IVU showed excretion of contrast by the right kidney. There was loss of cortical thickness with dilatation of the pelvicalyceal system consistent with chronic pyelonephritis. In May 1999, the IVU revealed a dilated right calyceal system with marked cortical thinning (Figure 2). In August 1999, MAG 3 renogram showed a single functioning right kidney demonstrating poor uptake of $2.9 \%$ at $2 \mathrm{~min}$. The excretion was slow. An ultrasound scan of the right kidney was performed in December 1999; the scan showed dilatation of the upper pole calyces with rather good overlying cortical thickness. However, there was loss of cortical thickness over the mid- and lower poles of the right kidney. A staghorn calculus was noted.

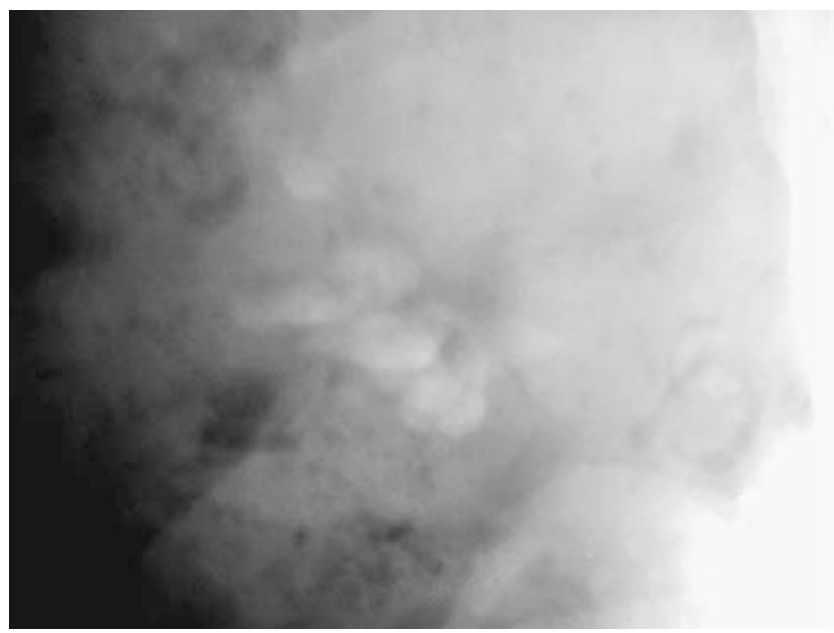

Figure 1 X-ray KUB of 28 March 1995 shows staghorn calculus in right kidney

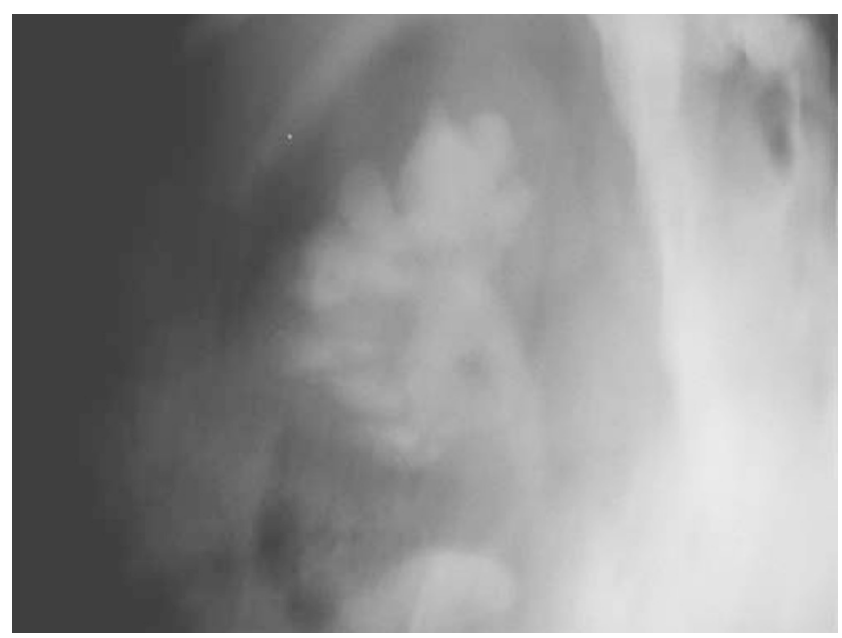

Figure 2 IVU of 18 May 1999 shows the right calyceal system dilated. There is marked cortical thinning

The patient was assessed in 2000 for pyelolithotomy. Her weight was $53.7 \mathrm{~kg}$. Blood pressure was $145 / 95$ $\mathrm{mmHg}$. The respiratory rate was 30 per minute at rest. The patient became dyspnoeic on mild exertion. She had no daytime somnolence. She would sleep on an air mattress with two pillows in a propped-up position. She was very short with a protuberant abdomen. She had a scar on the right side of the neck from a ventriculo-atrial shunt. She had a more extensive scar on the left side of the neck. Creatinine clearance was $18 \mathrm{ml} / \mathrm{min}$. Tidal volume: $150 \mathrm{ml}$. Vital capacity: $470 \mathrm{ml}$. The results of arterial blood gas analysis, which were performed on 20 March 2000, were as follows.

\begin{tabular}{lll}
\hline $\mathrm{pH}$ & 7.29 & $(7.32-7.45)$ \\
$\mathrm{pCO}_{2}$ & $4.4 \mathrm{kPa}$ & $(4.6-6.0)$ \\
$\mathrm{pO}_{2}$ & $11.8 \mathrm{kPa}$ & $(12.0-13.3)$ \\
Actual bicarbonate: & $16.2 \mathrm{mmol} / 1$ & $(20.0-32.0)$ \\
Base excess: & $-8.6 \mathrm{mmol} / 1$ & $(-2.0-2.0)$ \\
Standard bicarbonate: & $18.1 \mathrm{mmol} / 1$ & $(22.0-26.0)$
\end{tabular}

Comment: acute metabolic acidosis

The patient was considered unsuitable for anaesthesia for pyelolithotomy on account of the very high likelihood of difficulty in weaning off the ventilator after a surgical procedure, which is to be performed under general anaesthesia. There was almost no venous access; the usual subclavian and jugular veins were not accessible because of previous surgical procedures for the ventriculo-atrial shunt.

In June 2002, the patient's mother brought her to the spinal unit with a history of not passing urine for about $24 \mathrm{~h}$. An electrocardiogram showed sinus tachycardia, but no features attributable to hyperkalaemia. Urea: $14.7 \mathrm{mmol} / 1$; creatinine: $224 \mu / 1$; bicarbonate: $13 \mathrm{mmol} / 1$; sodium: $130 \mathrm{mmol} / 1$; potassium: $4.0 \mathrm{mmol} / 1$. The patient 
was given meropenem $500 \mathrm{mg}$ intravenously. Intravenous infusion of sodium bicarbonate $1.26 \%$ was started.

Ultrasound revealed moderate hydronephrosis with calculi in the right kidney. Right nephrostomy was planned. During the radiological procedure, the patient was turned prone from the supine position. The heart rate decreased to 44 and oxygen saturation dropped to $67 \%$ on air. The patient was given atropine sulphate $0.6 \mathrm{mg}$ intravenously. The heart rate increased to 110 and oxygen saturation rose to $99 \%$ while breathing air with 41 of added oxygen. A single puncture of the right kidney was performed and an 8-Fr, locking pigtail catheter was inserted. The radiological procedure took less than $10 \mathrm{~min}$ and was accomplished without anaesthesia or sedation. Following relief of urinary tract obstruction, there was postobstructive diuresis $(3765 \mathrm{ml} /$ $24 \mathrm{~h}$ ). At 2 weeks after nephrostomy, plasma creatinine was $125 \mu / 1$; urea: $5.2 \mathrm{mmol} / 1$.

Chest X-rays taken at 4, 6 and 11 days after nephrostomy showed clear lungs; but the lung volume was restricted due to kyphoscoliotic deformity of the vertebral column (Figure 3). A CT scan of the abdomen, which was performed 5 days after nephrostomy, showed the nephrostomy tube located in the proper position within the right kidney. There was still moderate right hydronephrosis (Figure 4). There was no evidence of any perinephric or other abdominal collection. The left kidney was not identified. A nephrostogram was performed 8 days after percutaneous nephrostomy. Calculi were seen in the pelvicalyceal system. There was some narrowing at the level of the pelvi-ureteric junction. However, contrast flowed freely into the right ureter and then into the ileal loop (Figure 5). This led us to conclude that anuria was the result from a ball-valve-type obstruction due to one of the calculi now lying freely in the renal pelvis. At 3 days after nephrostomy, crepitations were noted in the chest. In order to prevent pulmonary oedema, fluids were restricted (intake: $1404 \mathrm{ml}$; output: $2388 \mathrm{ml}$ ). At 17 days

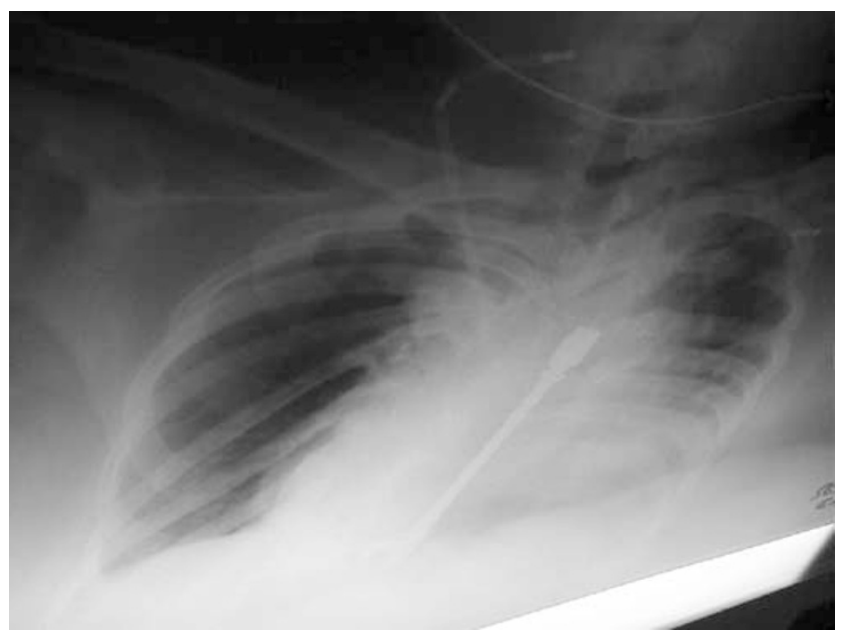

Figure 3 Chest X-ray, taken on 1 July 2002 (11 days after nephrostomy), shows clear lungs. The lung volume is restricted due to kyphoscoliosis

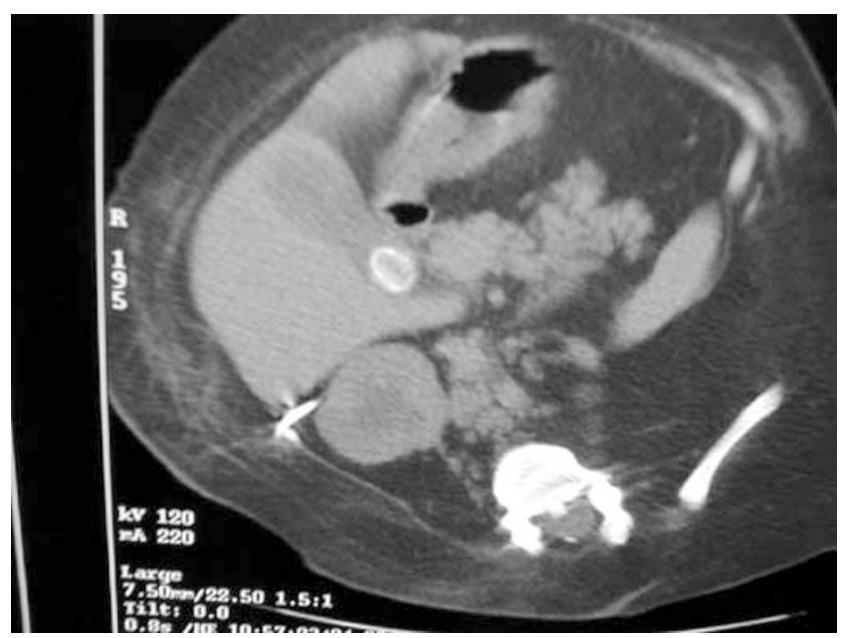

Figure 4 CT of abdomen, performed on 25 June 2002 (5 days after nephrostomy), shows that there is still moderate right hydronephrosis. A gallstone is seen in the gall bladder. The left kidney is not identified

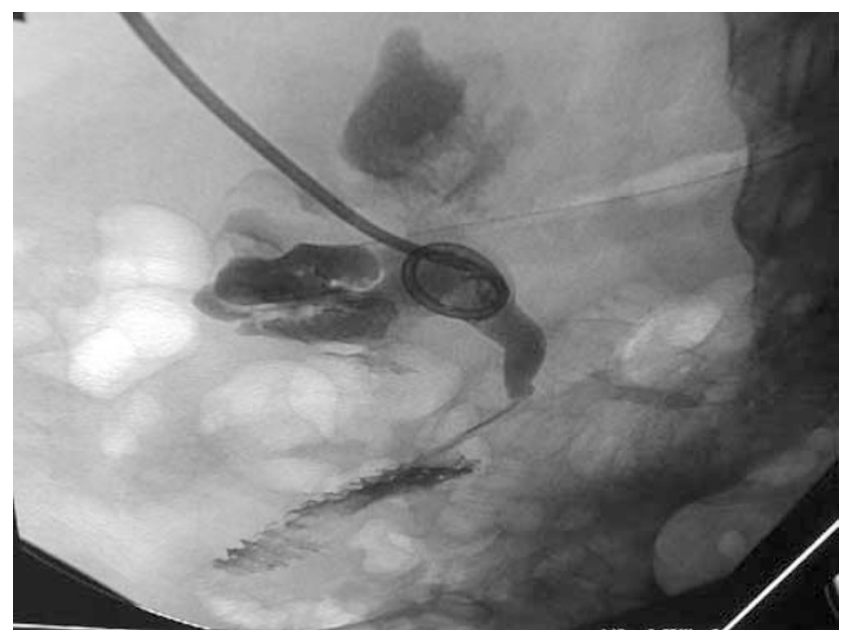

Figure 5 Nephrostogram, performed on 26 June 2002 (6 days after nephrostomy), shows some narrowing at the level of the pelvi-ureteric junction. However, the contrast flows freely into the right ureter and then into the ileal loop

after nephrostomy, the patient became very confused and agitated. Temperature was $35.9^{\circ} \mathrm{C}$. Oxygen saturation, while breathing $30 \%$ oxygen, was $93 \%$. She had very shallow and rapid breathing. Ronchi were audible over the chest. The next day, her clinical condition deteriorated further. While breathing $40 \%$ oxygen, oxygen saturation varied from 60 to $80 \%$. She became unresponsive to verbal command. She expired 19 days after nephrostomy.

\section{Discussion}

Regional Spinal Injuries Centre, Southport, UK

The general principle that staghorn calculus should not be left alone but surgery (percutaneous nephrolithotomy 
followed by shock wave lithotripsy of residual stone fragments) should be considered, may not be applicable to this patient with spina bifida, who had reached the age of 35 years. Our patient had severe kyphoscoliosis, chronic restrictive pulmonary function and almost no vascular access. We were concerned that it would indeed be difficult to wean this patient off the ventilator after a surgical procedure. In this patient, severe kyphoscoliosis with resultant pulmonary dysfunction and lack of vascular access presented insurmountable challenges for the surgical treatment of kidney stones.

\section{Professor Jean-Jacques Wyndaele}

This case is interesting as it permits one to understand several different aspects of urological and nonurological evolution in patients with a congenital spine tube defect.

Neural tube defects have prevalence in the population in UK of 7.8-8.4 per 10,000 for males and 9.0-9.4 per 10,000 females aged 10-69 years. ${ }^{4}$ Campbell et al, ${ }^{5}$ in their recent article, give a comprehensive overview of the characteristics of thoracic insufficiency syndrome associated with fused ribs and congenital scoliosis. The normal thorax is defined by two characteristics: normal, stable volume and the ability to change that volume. Volume depends on the width and depth of the rib cage, and the thoracic spine provides height. The ability to change volume (= thoracic function) is provided by the diaphragm and the secondary muscles of respiration. Thoracic insufficiency syndrome is the inability of the thorax to support normal respiration or lung growth. The condition of fused ribs and congenital scoliosis may result in a three-dimensional thoracic deformity with adverse effects on thoracic growth and function with development of thoracic insufficiency syndrome. On radiographs, the loss of the vertical height of the lung of the concave, restricted hemithorax is defined by the percentage of space available for the lung. Spine rotation causes a pathologic thorax, with restriction of the volume of the convex hemithorax and restriction of the motion of the involved ribs. Constrictive threedimensional deformity of the thorax may cause extrinsic, restrictive lung disease. This condition has led, in the patient described here, to the impossibility of obtaining the anaesthesia needed for the kidney operation.

The fate of children with myelomeningocoele has improved substantially during the last decades. Bowman et $a l,{ }^{6}$ in their 25 -year follow-up in 71 patients, found that at least $75 \%$ of children born with a myelomeningocoele can be expected to reach their early adult years. In total, $85 \%$ are attending or have graduated from high school and/or college. More than $80 \%$ of young adults have social bladder continence. However, complications are frequent. In the same study, $49 \%$ had scoliosis, with $43 \%$ eventually requiring a spinal fusion. In all, 16 patients $(23 \%)$ have had at least one seizure. Approximately one-third of patients are allergic to latex, with six patients having experienced a life-threatening reaction. Renal failure had a rate ratio of $6.8-9.0$ for male and
9.2-11.5 for female patients compared with the general population in each of the years 1994-1997. ${ }^{7}$ In those patients where bladder management is difficult, urinary diversion is sometimes unavoidable. As a more general technique for treating lower urinary tract disorders in these children, diversion surgery has not been advocated for many years already.

The described incidence of renal deterioration after conduit urinary intestinal diversion varies from 10 to $60 \%$. This variance is perhaps because many reports include both renal units that were abnormal and those that were normal before diversion. When the incidence of renal deterioration is determined by comparing renal units that were normal before diversion and then deteriorated postoperatively, $18 \%$ of patients who have ileal conduits show progressive deterioration. In the patients with ileal conduits, about $6 \%$ ultimately die of renal failure. ${ }^{8}$ Over the long term (20 years), $7 \%$ of patients have renal failure requiring dialysis, and $60 \%$ show deterioration of the upper tracts.

The electrolyte abnormality that mostly occurs with implantation of the ileum in the urinary tract is hyperchloraemic metabolic acidosis as in this patient. This acidosis occurs to some degree in most patients who have ileum interposed in the urinary tract, but is generally of a minor degree. Hyperchloraemic acidosis has been reported with a frequency of $68 \%$ (19 of 28 patients -10 of the 19 cases were severe enough to require treatment) in patients with ileal conduits. ${ }^{9}$ In another study, $70 \%$ of patients with ileal conduits followed for 4 years or more had a decreased serum bicarbonate level. ${ }^{10}$ Severe electrolyte disturbances occur to a much lesser degree in $10 \%$ of patients with ileal conduits. ${ }^{11}$ Symptoms in those in whom the syndrome is severe include easy fatigability, anorexia, weight loss, polydypsia, and lethargy.

The mechanism of hyperchloraemic metabolic acidosis is the ionised transport of ammonium. Ammonium substitutes for sodium in the $\mathrm{Na}^{+}-\mathrm{H}^{+}$antiport. The exchange of the weak acid $\mathrm{NH}_{4}^{+}$for a proton is coupled with the exchange of bicarbonate for chloride. Thus, ammonium chloride is absorbed across the lumen into the blood in exchange for carbonic acid (ie, $\mathrm{CO}_{2}$ and water). Ammonium may also gain entry to the blood from bowel lumen through potassium channels. ${ }^{12}$

Osteomalacia has been reported in patients with ileal conduits. The cause of osteomalacia may be multifactorial but commonly involves acidosis. With persistent acidosis, the bone calcium, released by the bone, buffers the excess protons. With its release, the kidney excretes the calcium. It has also been shown, however, that major alterations in serum bicarbonate levels are not necessary for the development of the syndrome. ${ }^{13}$ Moreover, some patients with osteomalacia secondary to urinary intestinal diversion do not have bony demineralisation corrected with restoration of normal acid-base balance. These patients have been found to manifest vitamin D resistance that is independent of the acidosis. Also, it has been shown that reabsorption of urinary solutes may play a role in increasing calcium 
excretion by the kidney. Sulphate filtered by the kidney inhibits calcium reabsorption and results in both calcium and magnesium loss by the kidney. Thus, if the gut increases its sulphate reabsorption and requires the kidney to increase sulphate excretion, this results in hypercalciuria and hypermagnesuria. ${ }^{14}$ The treatment of hyperchloraemic metabolic acidosis involves administering alkalising agents or blockers of chloride transport.

There is experimental evidence for impaired linear growth in urinary intestinal diversion. When followed over the long term, rats with unilateral ureterosigmoidostomy demonstrate significantly decreased femoral bone length compared with nondiverted controls. ${ }^{15}$ Thus, it appears clear that although obvious alterations in growth and development do not occur, when carefully studied, patients who have urinary intestinal diversions created in childhood and who maintain these diversions for more than 10 years have significant changes in linear growth.

The type of stone in this patient was most probably a struvite stone. Such a stone is composed of magnesium, ammonium, and phosphate, mixed with carbonate. Struvite calculi have also been commonly referred to as infection or triple-phosphate stones and account for $2-20 \%$ of all stones. ${ }^{16}$ Two conditions must coexist for the crystallisation of struvite $-\mathrm{a}$ urine $\mathrm{pH}$ of 7.2 or above and ammonia in the urine. ${ }^{17}$ The driving force behind struvite stones is infection of the urine with urease-producing bacteria. By far, the most common organism associated with struvite calculi is Proteus mirabilis. ${ }^{18}$ Struvite calculi account for the majority of staghorn stones observed in most countries. They can grow quite large and may fill the collecting system. Struvite stones may form on a nidus of calcium oxalate stones and grow quite rapidly. Most infection stones are radiopaque, but poorly mineralised matrix stones are faintly radiopaque or radiolucent. Two urologic conditions contribute to the tendency to form struvite calculia foreign body in the urinary tract and a neurogenic bladder. Patients with indwelling Foley catheters, urinary diversion, or lower tract voiding dysfunction are prone to developing these stones. ${ }^{15}$ Patients with staghorn calculi should not be followed up without treatment. The initial treatment is procedural to render the patient stone-free, followed by supportive medical therapy to prevent recurrent urinary tract infection. To cure infection stones, one needs to remove them completely. Most clinicians use a combination of percutaneous nephrolithotomy and ESWL for the treatment of patients with large calculi, particularly if many branches of the collecting system are involved. Long-term, culture-specific antimicrobials often reduce the bacterial burden, even if they do not completely sterilise the urine. Bacteria are still located on the surface or within the lattice of the stones, however, and thus reinfection is common with cessation of antibiotic therapy.

In this patient, a combination of several conditions led to the final outcome. Waiting to treat does not seem an option if treatment is possible at some stage.

\section{Dr Andrew Z Buczynski}

It was a very difficult situation for doctors to manage with the patient having so many congenital malformations such as myelomeningocoele with paraplegia, severe kyphoscoliosis, hydrocephalus with shunt, solitary kidney, and additionally extremely difficult vascular access. Supravesical urinary diversion was the only solution for the prevention of fast kidney damage. Unfortunately, stone formation in this solitary kidney occurs. Percutaneous nephrolithotomy (PCNL) in this case was the only solution. The question is why the patient developed the first and recurrent stone formation having an unobstructed kidney? It should be considered for two reasons: infection by urea-splitting microorganisms and/or obstruction at the level of the ileocutaneous junction. Some kind of obstruction could be considered because dilatation of the pelvicalyceal system was found on IVU since 1997 and was still present. We know that complete elimination of stones was performed during PCNL, but we do not know whether eradication of infection was successfully carried out. If it was impossible, urease inhibitors could be used to decrease urine $\mathrm{pH}$ and ammonia level. In those cases, methenamine hippurate (Urotractan) should be recommended for the prevention of recurrent infection and struvite calculosis in patients with neurogenic voiding dysfunction (NVD).

\section{Professor Eiji Iwatsubo}

This case seems to be the most complicated and difficult one for urologists to treat renal calculi. For surgical intervention, general status, indication for approach, and technical skill with good assessment of prognosis should be carefully considered. In spina bifida patients, skeletal anomaly often accompanies anomaly of urinary tract. Spina bifida with solitary kidney is not rare, since these anomalies are both of mesodermal origin. Highthoracic-level spina bifida must have had neuropathic bladder and bowel dysfunction. Urinary diversion with ileal conduit might have been chosen for urinary complication and leakage, although the age at surgery is not mentioned. Urolithiasis is not a rare complication in the patient with spina bifida and ileal conduit, which might have caused urinary stasis, calcium phosphate metabolism imbalance, and infection. We rather choose a temporary cystostomy catheter until they grow up to adolescence than an ileal conduit, if necessary, for urinary diversion in the case of neuropathic bladder at younger ages. A long-term cystostomy catheter would not prohibit future recovery of the bladder capacity. It does not result in irreversible fibrotic contracted bladder. Even if the surgery for kidney stone seemed to be successful, this case with the ileal conduit must have had much risk for recurrence of an infective struvite stone, which easily develops to the staghorn stone. In this paper, the level and severity of her paralysis are not clear; however, scoliosis due probably to paralysis might have caused thoraco-lumbar deformity, restricting movement of the rib cage, resulting 
respiratory insufficiency in adulthood, which prohibited general anaesthesia and surgical approach for the kidney.

I think the last effort of percutaneous nephrostomy to save this solitary kidney was the correct decision and mandatory; however, it was too late. The patient's general status did not accept this reasonable manoeuvre. It may be easy to criticise malpractice and failure of the treatment in a court of justice; however, as physicians we are always trying our best in front of the sick patient. I think the first surgical approach with ileal diversion to control neuropathic bladder dysfunction was not reasonable. It is already known that ileal diversion in childhood is contraindicated and I think ileal diversion or neo-bladder should not be used to replace neuropathic bladder in younger patients. Knowledge, experience, and personal skill covered by evidence-based medicine are the essence to achieve surgical and clinical success.

\section{Professor Manfred Stoehrer}

The course of the patient's condition described here is gloomy. I have contemplated which type of statement would be appropriate, and decided to give a fundamental and critical comment based on the progress of this case.

The patient was born in 1967; this was before intermittent catheterisation and anticholinergic medication had emerged as the treatment of choice for patients with neurogenic detrusor overactivity. The high pressure during voiding could be treated effectively at that time by alpha-blockers.

The development of the patient's urinary tract condition was inevitable with the treatment available in those days - urinary diversion by ileal conduit, chronic recurrent infections, urolithiasis. Had the patient been born 5-10 years later, the more effective treatment for high pressures during the filling phase by intermittent catheterisation and anticholinergics, also available for young children, might have changed significantly and positively the progress of the patient's urinary tract condition.

The basic pathophysiologic and therapeutic considerations are discussed extensively and comprehensively in Wyndaele's comment. With this patient's problems in the 1960s and early 1970s, it would be futile to consider whether preventive antibiotics or urine acidification might have positively changed the progress. Anyhow, since 1989 the physicians have only reacted on the patient's condition, which could not be arrested or slowed down, and failed to act proactively. This is always dissatisfying: as physicians, we should act and not react. I would not have hesitated to remove the stones that occurred in 1991, or at least the staghorn calculus detected in 1995. In particular for this patient with a solitary kidney, a rather aggressive therapy is justified under the 'all or none' maxim. With specific antibiotic treatment to suppress the incidence of urinary tract infection and possibly acidification of the urine to prevent crystallisation of struvite afterwards, the prognosis of this patient then might have been much better than the fatal situation described here. I think that the inadequate therapy in this patient right from the beginning of the treatment has contributed significantly to the final fatal outcome.

\section{Helmut Madersbacher and Reinhard Peschel}

This case report deals with a myelomeningocoele (MMC) patient, who died at the age of 35 years 19 days after a percutaneous nephrostomy was performed because of a ball-valve-type obstruction at the pelvicureteric junction caused by a staghorn calculus in her right solitary kidney, which drained into an ileum conduit performed years ago. The deleterious outcome should draw our attention to the following aspects.

The prerequisite for the preservation of renal function, also in myelodysplastic patients, is adequate urological care. The urological care should start at birth and should comprise the same therapeutic methods we apply to spinal-cord-injured patients:

- regular bladder emptying without hyperpressure, if necessary by clean intermittent catheterisation;

- pharmacotherapy to relax an overactive detrusor;

- a long-term low-dose infection prophylaxis has proved to be efficient, and these measures are able to avoid major surgery in the long term.

In the 1970s, in quite a number of centres all around the world, specialists were of the opinion that an early ileum conduit could solve the urological problem. However, nowadays, we know that the results did not meet with these expectations. Approximately $18 \%$ of all patients who had normal renal units before diversion showed progressive deterioration; $7 \%$ required haemodialysis and $6 \%$ died of renal failure. ${ }^{8}$ Stone formations in the kidneys - mostly struvite calculi - are not uncommon in these patients. The two prerequisites for struvite calculi formation - urine $\mathrm{pH}$ of 7.2 or higher and ammonia in the urine - are often fulfilled in these patients. The infection of urine with urease-producing bacteria $(P$. mirabilis) together with foreign bodies in the urinary tract (catheters) and neurogenic lower urinary tract dysfunctions as such predispose to this type of urinary infection. The current concept in the urological management of these children, which is based on conservative measurements (see above), has substantially improved the urological fate during the last two decades. Therefore, any type of urinary diversion with wet or continent stoma with major reconstructive surgery of the lower urinary tract should always be the last resort, if conservative therapy fails.

Another aspect is the management of stone disease in patients with neurogenic lower urinary tract dysfunction. Most of these stones are struvite stones, as it probably was the case in this young lady, and urinary tract infection is mostly the driving force behind them. Therefore, management comprises (1) an initial treatment to render the patient free of renal calculi, followed 
by (2) measures to prevent recurrent urinary infections. Long-term antimicrobial therapy, which is based on regular urine cultures, is able to reduce urinary infections.

With regard to this young lady, after percutaneous nephrolithotripsy in 1989, adequate prophylaxis would have been mandatory including antibiotic prophylaxis for at least 6 months in combination with lowering urine $\mathrm{pH}$. The same procedures would have been necessary in 1991 after detecting a recurrent stone disease. Probably, at that time, another intervention together with adequate prophylaxis could have prevented the development of a staghorn calculus with hydronephrosis as it was diagnosed in 1997, 1999 and in June 2002. Even in 1999 , another percutaneous nephrolithotripsy or extracorporeal shock wave lithotripsy would probably have been possible.

In the situation of June 2002, the percutaneous nephrostomy performed was the treatment of choice to relieve urinary tract obstruction. However, at that time, comorbidity (severe kyphoscoliosis, chronic restrictive pulmonary function, and almost no vascular access), which obviously deteriorated over the years, caused the death of the patient.

To summarise, the clinical history of this patient with spina bifida, who died at the age of 36 years, clearly shows that any type of urinary diversion in these children should only be performed if all adequate conservative measures have failed. If calculi are formed, they are mostly of infectious origin, and the management required would be complete stone removal, and adequate prophylaxis including long-term antimicrobials and lowering the urine $\mathrm{pH}$ below 7.2.

\section{References}

1 Raj GV et al. The incidence of nephrolithiasis in patients with spinal neural tube defects. J Urol 1999; 162: 12381242.

2 Rege PR, Levine MS, Oppenheimer S, Evans AT. Renal calculi and biochemical abnormalities in children with myelomeningocele and ileoconduit diversion. Urology 1975; 5: 12-16.
3 Leweis MA, Webb NJ, Stellman-Ward GR, Bannister CM. Investigative techniques and renal parenchymal damage in children with spina bifida. Eur J Pediatr Surg 1994; 4 (Suppl 1): 29-31.

4 Lawrenson $\mathrm{R}$ et al. A UK general practice database study of prevalence and mortality of people with neural tube defects. Clin Rehab 2000; 14: 627-630.

5 Campbell Jr RM et al. The characteristics of thoracic insufficiency syndrome associated with fused ribs and congenital scoliosis. J Bone Joint Surg Am 2003; 85: 399-408.

6 Bowman RM et al. Spina bifida outcome: a 25-year prospective. Pediatr Neurosurg 2001; 34: 114-120.

7 Lawrenson $\mathrm{R}$ et al. Renal failure in patients with neurogenic lower urinary tract dysfunction. Neuroepidemiology 2001; 20: 138-143.

8 Richie JP, Skinner DG, Waisman J. The effect of reflux on the development of pyelonephritis in urinary diversion: an experimental study. J Surg Res 1974; 16: 256-261.

9 Castro JE, Ram MD. Electrolyte imbalance following ileal urinary diversion. Br J Urol 1970; 42: 29-32.

10 Malek RS, Burke EC, DeWeerd JH. Ileal conduit urinary diversion in children. J Urol 1971; 105: 892-900.

11 Schmidt JD, Hawtrey CE, Flocks RH, Culp DA. Complications, results, and problems of ileal conduit diversions. J Urol 1973; 109: 210-216.

12 Koch MO, McDougal WS. The pathophysiology of hyperchloremic metabolic acidosis after urinary diversion through intestinal segments. Surgery 1985; 98: 561-570.

13 Koch MO, McDougal WS. Bone demineralization following ureterosigmoid anastomosis: an experimental study in rats. J Urol 1988; 140: 856-859.

14 McDougal WS, Koch MO. Effect of sulfate on calcium and magnesium homeostasis following urinary diversion. Kidney 1989; 35: 105-115.

15 Herring LC. Observations of 10,000 urinary calculi. J Urol 1962; 88: 545.

16 Nemoy NJ, Stamey TA. Surgical, bacteriological, and biochemical management of infection stones. JAMA 1971; 215: 1470-1476.

17 Silverman DE, Stamey TA. Management of infection stones: the Stanford experience. Medicine (Baltimore) 1983; 62: 44-51.

18 Comarr AE, Kawaichi GR, Bors E. Renal calculosis in patients with traumatic cord lesions. J Urol 1962; 85: 647-656. 